\title{
A method research based on acceleration unbalance power fast estimation of generator tripping sensitive spot search
}

\author{
Liu Ying ${ }^{\mathrm{a}}$, Xu Wei-ting ${ }^{\mathrm{b}}$, Tang Quan, Feng Han \\ (State Grid Sichuan Economic Research Institute, Chengdu ,610041, Sichuan Province, China) \\ aemail: 281641488@qq.com, bemail: mailtott@163.com
}

Keywords: Energy Function; Unbalance Power; Superposition Principle; Sensitive Points

\begin{abstract}
The generation tripping sensitive point could be located by absolute kinetic energy incremental index. A method was proposed which is used to locate this point based on the estimation of unbalance power during short circuit period. The voltage was separated to two parts: one was determined by the pre-fault power flow, the other was determined by the equivalent voltage at the fault point. The voltages of the generators were calculated with superposition principle. Then the unbalance power of generators could be computed. The sensitive points of generation tripping were determined by this power. The effectiveness of the proposed approach is verified by simulation results of a ten-machine 39-bus system.
\end{abstract}

\section{Introduction}

Generator tripping and load shedding are the most widely used and mature emergency control measures in power system. Considerable research effort has been devoted to the methods. The Extend Equal Area Criterion(EEAC) is used for fast valving and generator tripping[1]. On the basis of local energy function (PEF), maximum speed of generator is testing with PMU and can be used to determine the amount of generator tripping after compare with the threshold value[2,3]. In [4], a emergency control optimal model which combined with the configuration and operation requirements of the actual power grid security and stability control system and under transient stability based on the perturbed trajectories (results from time domain simulation) is provided, one method of generator tripping is depends on the amount of unbalance power. $\Delta \mathrm{P}$ integral is the start-up criterion of transient stability control[5]. In [6], the concept of absolute kinetic energy increment(AKEI) is proposed and it can be supplied in identifying Instability Model. The emergency control under $0-1$ programming can be utilized on the ground of absolute kinetic energy increment (AKEI)[7].

In this paper, a fast estimation based on generator's unbalance power is proposed. The voltage was separated to two parts, the voltage and phase angle during short circuit period was calculated with superposition principle. Then the unbalance power of generators could be computed, and the sensitive points of generation tripping can be determined by the power.

\section{Research on system instability sensitivity index during short circuit period}

For the study, simplify the power system: synchronous generator is expressed as vertical axis transient reactance $x_{d}^{\prime}$ and a constant potential, the voltage phase angle is keeping with the rotor angle $\delta$ phase, using constant impedance load modeling, the input mechanical power $P_{m i}$ is assumed to be constant. Based on the above assumptions, the differential equation for multi-machine power system dynamic characteristics is:

$$
\begin{aligned}
& M_{i} \frac{d^{2} \delta_{i}}{d t^{2}}=-D_{i} \frac{d \delta_{i}}{d t}+P_{m i}-P_{e i} \\
& (i=1,2, \cdots, n)
\end{aligned}
$$


Where

$$
P_{e i}=E_{i}^{2} g_{i i}+\sum_{\substack{j=1 \\ j \neq i}}^{n}\left[C_{i j} \sin \left(\delta_{i}-\delta_{j}\right)+D_{i j} \cos \left(\delta_{i}-\delta_{j}\right)\right]
$$

Suppose

$$
\begin{gathered}
\frac{d^{2} \delta_{i}}{d t^{2}}=\ddot{\delta}_{i}, \frac{d \delta_{i}}{d t}=\dot{\delta}_{i}, P_{m i}-E_{i}^{2} g_{i i}=P_{0 i} \\
\sum_{\substack{j=1 \\
j \neq i}}^{n}\left[C_{i j} \sin \left(\delta_{i}-\delta_{j}\right)+D_{i j} \cos \left(\delta_{i}-\delta_{j}\right)\right]=P_{i}
\end{gathered}
$$

Equation 1 can be impressed as:

$$
M_{i} \ddot{\delta}_{i}=-D_{i} \dot{\delta}_{i}+P_{0 i}-P_{i}
$$

The phase Angle at central defined by:

$$
\delta_{0 i}=\frac{1}{M_{T}} \sum_{i=1}^{n} M_{i} \delta_{i} M_{T}=\sum_{i=1}^{n} M_{i}, \theta_{i}=\delta_{i}-\delta_{0}, \omega_{0}=\dot{\delta}_{0}, \dot{\theta}_{i}=\omega=\omega-\omega_{0} \text {, considering zero damping }
$$

situation in center of inertia (COI), the system motion equation is:

$$
\left\{\begin{array}{l}
M_{T} \ddot{\delta}_{0}=P_{C O I} \\
M_{i} \dot{\omega}=P_{i 0}-P_{i}-\frac{M_{i}}{M_{T}} P_{C O I} \\
\dot{\theta}_{i}=\omega
\end{array}\right.
$$

Where

$$
P_{\text {COI }}=\sum P_{0 i}-2 \sum_{i=1}^{n-1} \sum_{j=i+1}^{n} D_{i j} \cos \delta_{i j}
$$

For the i th machine, it can be expressed by the partial energy function (PEF)[2][5]:

$$
\begin{aligned}
& V_{i}(\theta, \omega)=\frac{1}{2} M_{i} \omega_{i}^{2}-P_{0 i}\left(\theta_{i}-\theta_{i}^{t}\right)- \\
& \sum_{\substack{j=1 \\
\neq i}}^{n} \frac{\theta_{i}-\theta_{i}^{t}}{\theta_{i j}-\theta_{i j}^{t}}\left[C _ { i j } \left(\cos \theta_{i j}-\cos \theta_{i j}^{t}-\right.\right. \\
& \left.D_{i j}\left(\sin \theta_{i j}-\sin \theta_{i j}^{t}\right)\right]+\frac{M_{i}}{M_{T}}\left[\sum_{k=1}^{n} P_{k}\left(\theta_{i}-\theta_{i j}^{t}\right)-\right. \\
& \left.2 \sum_{k=1}^{n-1} \sum_{j=k+1}^{n} \frac{\theta_{i}-\theta_{i}^{t}}{\theta_{i j}-\theta_{i j}^{t}} D_{k j}\left(\sin \theta_{k j}-\sin \theta_{k j}^{t}\right)\right]
\end{aligned}
$$

In general, time from the short circuit fault occurs to fault clears is relatively short. Although the generator rotor's speed changes sharply but the distance between the rotor of different generators is not space out during this time, and the change of the phase angle relative to center of inertia is not obvious. Consider the above, the potential energy form of the partial energy function can be ignored so the PEF can be expressed as:

$$
V_{i} \approx \frac{1}{2} M_{i} \omega_{i}^{2}
$$

Then discuss the relation between PEF and the unbalance power of generator during outage. The $i$ th generator's swing equation of the generator rotor relative to center of inertia is:

$$
M_{i} \dot{\omega}_{i}=P_{m i}-P_{i}-\frac{M_{i}}{M_{T}} P_{C O I}
$$

For each generator in power system, its inertia is much less than the inertia of the whole system, $M_{i}<<M_{T}$, considering it we get (7): 
Combining (7) with (5), the PEF becomes:

$V_{i}=\frac{1}{2 M_{i}}\left|\int_{0}^{t} \Delta P_{i} d t\right|^{2}$

Where

$$
\Delta P_{i}=P_{m i}-P_{i}
$$

We can conclude from (8) that the critical variables is $\left.\int_{0}^{t} \Delta P_{i} d t\right|^{2}$, Its represents the acceleration $\Delta \omega$ of the unit and reflect the unit's accelerate energy after fault.

Research shows that, the absolute kinetic energy increment index in short circuit period display a substantial correlation with generator instability mode[6]. The generator out of step at first has more kinetic energy increment than the rest. We make use of the absolute kinetic energy increment in short circuit period as criteria for judging the instability mode, it is obtained as:

$$
\Delta E_{i k}=\int_{0}^{t_{c}} \Delta P_{i} d t
$$

The reason why using the absolute kinetic energy increment $\triangle$ Eik instead of PEF as sensibility control index is that we can get whether the generator is acceleration or deceleration by the $\triangle$ Eik 'value (positive or negative). On the contrary, the PEF kinetic energy index is always positive value no matter the generator is acceleration or deceleration. Absolute kinetic energy increment with the clear physical definition: the actual mechanical kinetic energy increment of generator rotor in the integral time.

In equation (9), tc is the clearing time. Assume $\Delta P_{i}$ is a constant during the failure period, equation (9) can be simplified as the approximate absolute kinetic energy increment:

$$
\Delta E_{i k}=\Delta P_{i} \times t_{c}
$$

The assumption based on the condition that the short circuit fault duration is very short. To the power system model which contraction in the generator node and consisted of $\mathrm{n}$ generators, the electric power of generator is:

$$
\begin{aligned}
& P_{i}=E_{i}^{2} G_{i i}+ \\
& \sum_{\substack{j=i \\
\neq i}}^{n}\left(E_{i} E_{j} B_{i j} \sin \delta_{i j}+E_{i} E_{j} G_{i j} \cos \delta_{i j}\right)
\end{aligned}
$$

According to the analysis above, use approximate absolute kinetic energy increment of generator ( $\Delta E_{i k}=\Delta P_{i} \times t_{c}$ ) as sensibility control index. To calculate the index, we need a special power flow algorithm for the calculation of unbalance power during the short circuit period.

\section{Research based on superposition principle of accelerating power fast estimation}

The short circuit fault cause energy blockage in the generator. The absolute kinetic energy increment of generator in short circuit period can be obtained by integrating the accelerating power, the kinetic energy increment has great impact on instability mode. Although we can get accurate kinetic energy increment through digital simulation, but the digital simulation is time consuming and difficult to conduct a quick scan of expected failure in a short period of time. In this section, a short circuit current calculation method based on superposition principle is proposed, according to the accelerating power fast estimation of generator during failure period, the absolute kinetic energy increment can be estimated.

The power flow in three-phase short circuit period can't be calculated by traditional power flow algorithms: Newton Laphson iteration, PQ decoupled load flow. Notice the two points:

(1) the voltage of short circuit node drop to zero;

(2)the voltage of generator's inner points is constant.

So the power flow in short circuit period can be calculated by a method based on superposition 
principle.

By means of the principle of superposition, the network state in failure state is divided into two states[8], one is the steady state before fault occurs, shown as figure1(a). In another state, as figure1(b), the generator voltage is zero, plus one potential of the fault point $\mathrm{D}$, the potential is of the same magnitude but opposite polarity as the $\dot{V}_{d}, \dot{V}_{d}$ is the voltage of point $\mathrm{D}$ in normal condition.

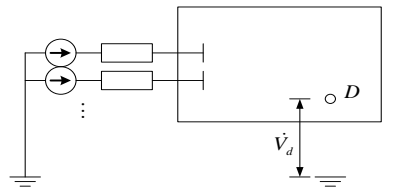

(a)

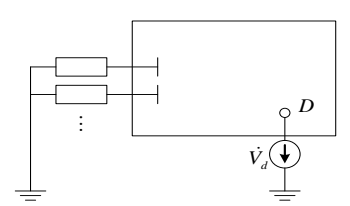

(b)

Fig. 1. Application of Superposition principle

With the superposition of the two states, the voltage of fault point D is zero in single-phase system, it is equal to the three-phase short circuit state. The voltages of each node calculated in the second state is the fault component of equivalent potential of failure point during short circuit period. Superimpose the result on the first state, we get the node voltage during short circuit period.

When calculate the node voltage during short circuit period based on superposition principle. The voltage of fault point before fault occurs is $\dot{V}_{d}^{(0)}$, with self-impedance Zdd of the fault point D, the short circuit current is:

$$
\dot{I}_{d}=-\frac{\dot{V}_{d}^{(0)}}{Z_{d d}}
$$

Conventionally, the positive direction of electric current is that of the injection node current, consequently, get rid of negative value of equation (12), combining short circuit current as the only nonzero element with network equation:

$$
\mathbf{Z} \cdot \dot{\mathbf{I}}=\dot{\mathbf{V}}
$$

The component of voltage of each node arise from short circuit current is:

$$
\dot{V}_{i(d)}=-\frac{\dot{V}_{d}^{(0)}}{Z_{d d}} Z_{d i} \quad(i=1,2, \cdots, n)
$$

Where Zdi is the mutual impedance between short point $\mathrm{D}$ and node $\mathrm{i}$. the node voltage during short circuit period can be obtained by adding the component of voltage to the node voltage $\dot{V}_{i}^{(0)}$ before failure:

$$
\dot{V}_{i}=V_{i} \angle \theta_{i}=\dot{V}_{i}^{(0)}+\dot{V}_{i(d)}
$$

According to the above method, calculate the generator terminal voltage during failure period and estimate the accelerating power, generator using the second-order model with $E^{\prime}$ is constant, on the basis of steady state power flow, generator internal potential before failure is:

$$
\dot{E}_{i}=E_{i} \angle \delta_{i}=\dot{V}_{i}+j X_{i} \dot{I}_{i}
$$

Steady state current $\dot{I}_{i}$ can be represented as:

$$
\dot{I}_{i}=\frac{P_{i}-j Q_{i}}{\dot{V}_{i}^{*}}
$$

$\mathrm{Pi}$ and Qi is respectively: active power and reactive power, according to the fact that the power-angle won't mutate when fault taking place and $E^{\prime}$ is constant, we can estimate the electric power of generator during failure time:

$$
P_{i s}=\frac{E_{i} V_{i}}{X_{d i}} \sin \left(\delta_{i}-\theta_{i}\right)
$$


Assuming that the mechanical power keeps constant in short circuit period, then the unbalance power is:

$$
\Delta P_{i}=P_{i s}-P_{i}
$$

By above research, the position of sensitive control point can be confirmed according to the amount of unbalance acceleration power during short circuit period, it is beneficial to deciding the position and amount of generator tripping.

\section{Realization of unbalance power rapid estimation algorithm}

\subsection{The processing of load and generator branch}

The method for estimating unbalance power is proposed in above section, some problems remains to be solved to realize the algorithm. The first step is processing the load, convert load into impedance, substituting it in nodal admittance matrix. Assuming that a power system consist of $\mathrm{N}$ nodes, there are $n$ generator nodes(node 1 to node $n$ ), nodes $n+1 \sim N$ are other nodes, the impedance corresponding to the load is:

$$
y_{l i}=\frac{P_{l i}-j Q_{l i}}{\left|V_{i}\right|^{2}}, \quad i=n+1, \cdots, n+N
$$

Where $P_{l i}+j Q_{l j}$ is the load of node $\mathrm{i},\left|V_{i}\right|$ is the voltage magnitude of node $\mathrm{i}$, dispose the generator node in the same way, the admittance of generator branch is expressed in the equation:

$$
y_{g i}=1 /\left(R_{i}+j x_{d i}{ }^{\prime}\right), \quad i=1,2, \cdots, n
$$

The node admittance matrix of the network is shown as equation (22):

$$
\mathbf{Y}_{\text {BUS }}=\left[\begin{array}{ll}
\mathbf{Y}_{1} & \mathbf{Y}_{2} \\
\mathbf{Y}_{3} & \mathbf{Y}_{4}
\end{array}\right] \quad \begin{gathered}
n-n \\
N-n
\end{gathered}
$$

The new node admittance matrix can be obtained by integrating with load and the motor branch:

$$
\mathbf{Y}_{\mathbf{D}}=\left[\begin{array}{cc}
\mathbf{y}+\mathbf{Y}_{1}+\mathbf{y}_{\mathrm{lg}} & \mathbf{Y}_{2} \\
\mathbf{Y}_{3} & \mathbf{Y}_{4}+\mathbf{y}_{\mathrm{ll}}
\end{array}\right] \begin{gathered}
n \\
N-n
\end{gathered}
$$

where

$$
\begin{gathered}
\mathbf{y}_{\mathrm{lg}}=\operatorname{Diag}\left(y_{g i}\right), \quad i=1, \cdots, n \\
\mathbf{y}_{\mathrm{ll}}=\operatorname{Diag}\left(y_{l i}\right), \quad i=n+1, \cdots, N
\end{gathered}
$$

With matrix inversion, the admittance matrix is:

$$
\mathbf{Z}_{\mathbf{D}}=\mathbf{Y}_{\mathbf{D}}^{-1}
$$

\subsection{Algorithm process}

The algorithm flow of accelerating power rapid estimation is shown in figure 2. 


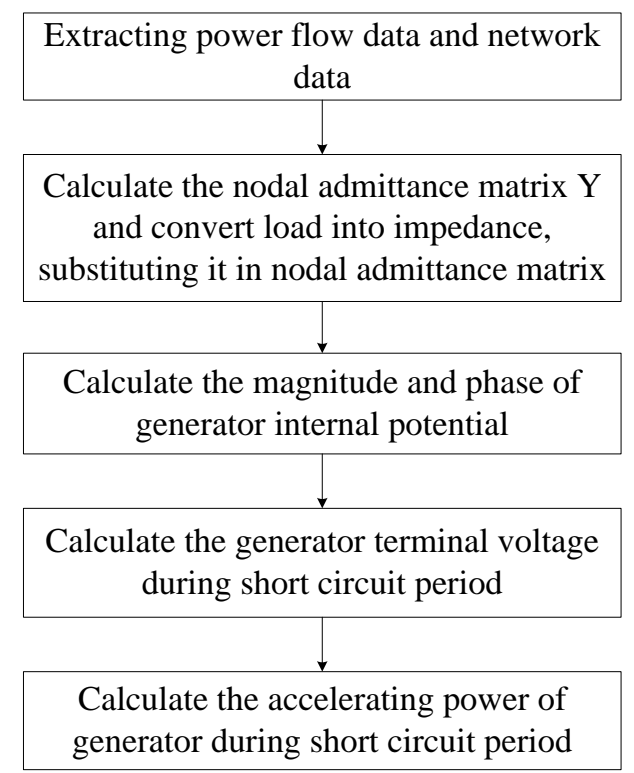

Fig. 2. Algorithmic process

\section{Analysis of examples}

In order to test the method we proposed, apply the 10-generator 39-bus system for simulation analyses. As shown in figure 3, assume three phase short circuit fault occurring on the bus 16, fault clearing for line $16-17$ is operated in 0.12 s later.

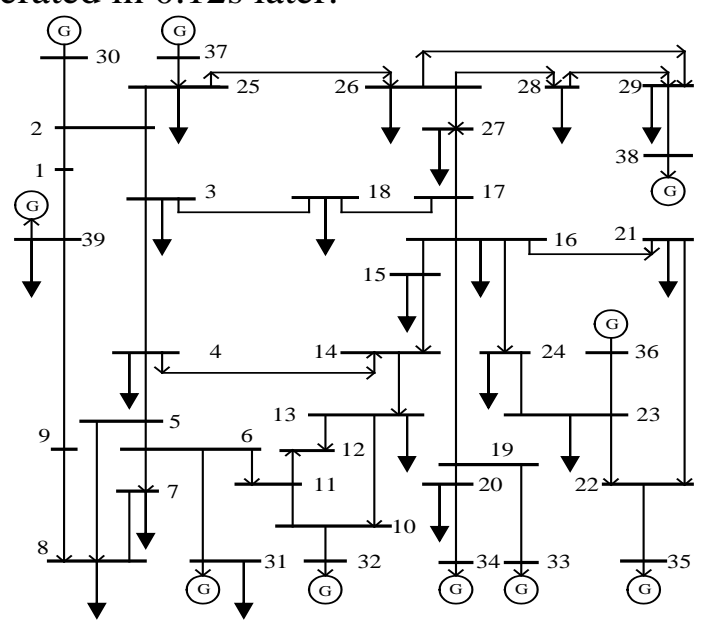

Fig. 3. 10-generator 39-bus system

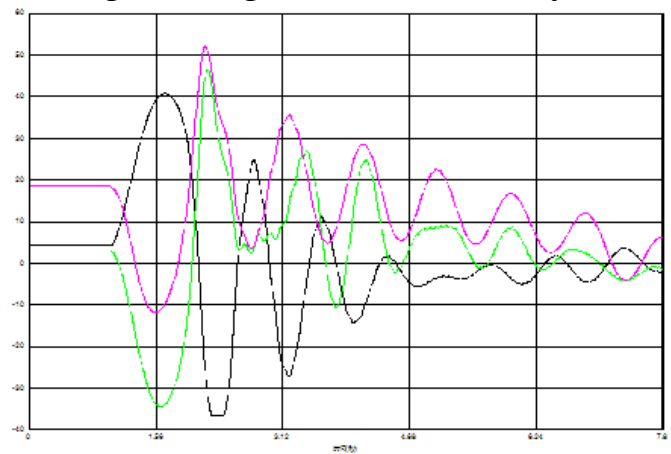

Fig. 4. Angle curve without emergency controls

If the emergency control is not carried out, the system will loss synchronization at first swing. The Angle curve as shown in figure 4. Apply the method proposed above for calculating accelerating power, the accelerating power of each generator is obtained as table 1 . 
Tab. 1. The unbalance power when short circuit occurs at bus 16

\begin{tabular}{llllll}
$\Delta P(\mathrm{MW})$ & 416 & 271 & 367 & 579 & 407 \\
\hline bus & 35 & 36 & 37 & 38 & 39 \\
$\Delta P(\mathrm{MW})$ & 618 & 497 & 245 & 416 & 142
\end{tabular}

As shown in table 1, the three bus of maximum accelerating power are: bus 33, bus 35 and bus 36. Determine the generator tripping control sensitive point is located in the three bus. Conduct generator tripping at bus 35 and bus 36, the generator tripping value for each bus is $100 \mathrm{MW}$ respectively, the system is stable, The Angle curve is shown in figure 5.

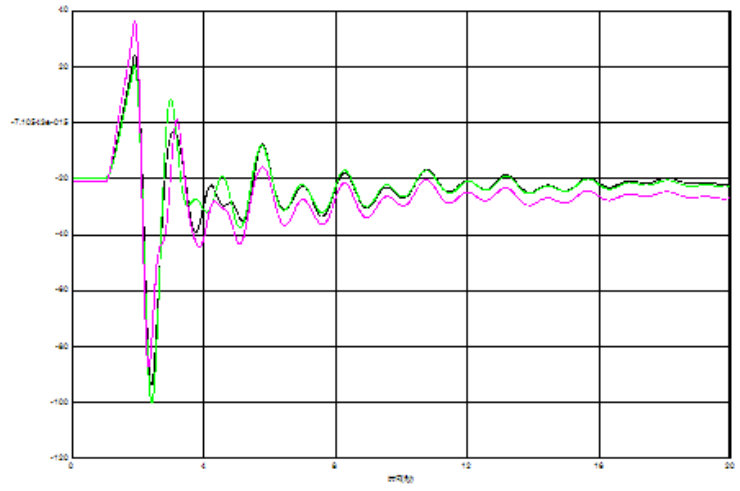

Fig. 5 .Angle curve with emergency controls

\section{Conclusion}

In this paper, an algorithm for computing unbalance power during short circuit is performed. Based on the superposition principle, assume the voltage in fault time consists of two parts: one is the voltage in normal condition, another voltage is produced by a voltage source which located in the failure point and its magnitude and phase angle are same with the voltage determined by the pre-fault power flow at fault point, but the voltage source has opposite polarity as the voltage at fault point under normal condition. We can get the unbalance power of generator after the calculating of generator voltage, the generator tripping control sensitive point can be determined by the unbalance power. Simulation on the 10-generator 39-bus system testifies that the method is correct and effective.

\section{References}

[1] Xue Yusheng, Li Juan, Mao Xueyan, Incorporation of fast valving and generator tripping in extended equal area criterion[J]. Automation of Electric Power Systems, 1993, 17(12)14-21.

[2] Stanton S E, Dykas W P. Analysis of a local transient control action by partial energy functions[J]. Power Systems, IEEE Transactions on, 1989, 4(3) 996-1002.

[3] Stanton S E, Slivinsky C, Martin K, et al., Application of phasor measurements and partial energy analysis in stabilizing large disturbances[J]. Power Systems, IEEE Transactions on, 1995, 10(1)297-306.

[4] Ren Wei, Fang Dazhong, Chen Jiarong, Optimal Control Theory Based Power System Emergency Control and Its Application[J]. Power System Technology, 2009, 33(2)8-13.

[5] Li Han, Wu Jingtao, Cui Wenjin, Study on the $\Delta \mathrm{P}$ integration strategy for generation shedding $[\mathrm{J}]$. Journal of Tsinghua University (Sci \&Tech), 1997, 37(7)93-96.

[6] Min Yong, Li Han, Lin Zifeng, Absolute kinetic energy increment of the generator rotor for distinguishing unstable modes[J]. Journal of Tsinghua University (Sci \&Tech), 2003, 43(9)1161-1164.

[7] Min Yong, Li Han, Lin Zifeng, Extended 0-1 programming on-line quasi real-time decision-making algorithm based on the absolute kinetic energy increment[J]. Proceedings of the CSEE, 2003, 23(3) 5-9. 
[8] Xi`an Jiaotong University, Tsinghua University, Zhejiang University. Power system computation[M]. Water Conservancy and Electric Power Press.

[9] Fang Yongjie, Fan Wentao, Chen Yonghong, et al., An on-line transient stability control system of large power systems[J]. Automation of Electric Power Systems, 1999, 23(1)8-11.

[10] Zhu Chuanjiang, Xue Yusheng, Study on the phenomenon of isolated stability domain in transient stability assessmient[J]. Automation of Electric Power Systems, 1997, 21(2)27-31.

[11] Tan Bin, Xue Yusheng, A study on chaos of multi-machine systems[J]. Automation of Electric Power Systems, 2001, 25(2)3-8.

[12] Guo Qi, Zhao Jinquan, Zhang Boming, A study on isolated stability domain phenomenon in transient stability assessment based on OMIB[J]. Automation of Electric Power Systems, 2005, 29(19)14-18.

[13] Fang Yongjie, Application of emergency control to reduce risk of system collapse triggered by power transmission interface tripping: thinking on the India power blackouts[J]. Automation of Electric Power Systems, 2013, 37(4)1-5.

[14] Tang Yong, Bu Guangquan, Yi Jun, Analysis and lessons of the blackout in Indian power grid on July 30 and 31, 2012[J]. Proceedings of the CSEE, 2012, 32(25)167-174.

[15] Li Bijun, Xu Jianbing, Xu Taishan, et al., Engineering application of integrated and coordinated defense technology of large power system security and stability[J]. Automation of Electric Power Systems, 2008, 32(25)25-3 